\title{
Development and Validation of 3-Month Major Post-Stroke Depression Prediction Nomogram After Acute Ischemic Stroke Onset
}

Guo Li'
Ping Jing (D)
Guohua Chen
Junhua Mei
Jinfeng Miao'
Wenzhe Sun'
Yan Lan iD
Xin Zhao'
Xiuli Qiu'
Zhou Zhu',*
Suiqiang Zhu',*
'Department of Neurology, Tongji
Hospital, Tongji Medical College,
Huazhong University of Science and
Technology, Wuhan, Hubei, 430030,
People's Republic of China; ${ }^{2}$ Department
of Neurology, Wuhan Central Hospital,
Wuhan, Hubei, 4300I4, People's Republic
of China; ${ }^{3}$ Department of Neurology,
Wuhan First Hospital, Wuhan, Hubei,
430022, People's Republic of China

*These authors contributed equally to this work
Purpose: The early detection of major post-stroke depression (PSD) is essential to optimize patient care. A major PSD prediction tool needs to be developed and validated for early screening of major PSD patients.

Patients and Methods: A total of 639 acute ischemic stroke (AIS) patients from three hospitals were consecutively recruited and completed a 3-month follow-up. Sociodemographic, clinical and laboratory test data were collected on admission. With major depression criteria being met in the DSM-V, 17-item Hamilton Rating Scale For Depression (HRSD) score $\geq 17$ at 3 months after stroke onset was regarded as the primary endpoint. Multiple imputation was used to substitute the missing values and multivariable logistic regression model was fitted to determine associated factors with a bootstrap backward selection process. The nomogram was constructed based on the regression coefficients of the associated factors. Performance of the nomogram was assessed by discrimination (C-statistics) and calibration curve.

Results: A total of $7.04 \%$ (45/639) of patients were diagnosed with major PSD at 3 months. The final logistic regression model included age, baseline NIHSS and mRS scores, educational level, calcium-phosphorus product, history of hypertension and atrial fibrillation. The model had acceptable discrimination, based on a C-statistic of 0.81 (95\% CI, 0.791-0.829), with $71.1 \%$ sensitivity and $78.6 \%$ specificity. We also transformed the model to a nomogram, an easy-to-use clinical tool which could be used to facilitate the early screening of major PSD patients at 3 months.

Conclusion: We identified several associated factors of major PSD at 3 months and constructed a convenient nomogram to guide follow-up and aid accurate prognostic assessment.

Keywords: major post-stroke depression, nomogram, calcium-phosphorus product, C-statistic

\section{Introduction}

At the end of 2016, the life time risk of stroke in men and women was $24.7 \%$ and $25.1 \%$ globally, while the lifetime risk of stroke in Chinese men and women was $41.1 \%$ and $36.7 \%{ }^{1}$ Post-stroke depression (PSD) is recognized as one of the most common and severe neuropsychiatric sequelae of stroke, affecting about $40 \%$ of stroke patients. ${ }^{2,3}$ Since China is undergoing a rapid demographic transition and "super" aging, with a high incidence of stroke, the incidence of PSD will probably increase steadily in the next decades. A considerable number of studies have
Correspondence: Zhou Zhu

Tel +86-18I7I 081029

Email zhouzhu@hust.edu.cn

Suiqiang Zhu

Tel +86-|3035I01 |4|

Email zhusuiqiang@I63.com 
suggested that PSD, especially major PSD, was associated with worse neurological functional recovery, low quality of life (QoL), higher risk for cognitive impairment, severe impairment in social functioning, and increased mortality. ${ }^{4,5}$

Symptom remission of PSD over the first few months after stroke, which has been associated with higher recovery of the activity of daily living function for patients, is vital. ${ }^{6}$ Studies have shown that the prevalence of PSD varies at different time points, there exists a trend of rising firstly and then falling in the first year, and the highest incidence of depression falls in the first few months following stroke. ${ }^{7,8}$ PSD at 3 months after onset was highly predictive of disability at 5-year follow-up based on a national survey in China. ${ }^{9}$ A previous study has also demonstrated that the patients who were in the state of 3-month PSD would be more likely to have more severe functional impairment than patients who recovered from their depressive symptoms. ${ }^{10}$ However, 3-month PSD patients could not be diagnosed timely because caregivers of stroke patients often fail to notice the abnormal mood changes after hospital discharge. Hence, it is essential to explore associated factors and construct applicable predictive instruments to assist clinicians in early identification of patients at risk of 3-month major PSD.

Although PSD has been generally considered as a result of joint action of multiple mechanisms, ${ }^{11}$ the underlying pathophysiological mechanisms of PSD have remained unknown. ${ }^{12}$ Rapidly measurable biomarkers to predict PSD are pivotal for allocating healthcare resources and optimizing care. ${ }^{13}$ Recently published articles have revealed some predictive biomarkers for PSD. ${ }^{14-17}$ However, these biomarkers had poor accessibility in clinical practice, such as growth differentiation factor- 15 (GDF-15), antiphospholipid antibodies, irisin and serum neurofilament light (sNfL) levels. The preferable scenario is that we can obtain the effective biomarkers easily and without extra costs. ${ }^{18}$

Nomograms have been widely used as a reliable clinical tool to create a simple intuitive graph for quantifying the risk of a clinical event of interest, based on multiple logistic regression or Cox Proportional Hazards regression model. ${ }^{19,20}$ In this study, we aimed to find the clinical predictors and easily available biomarkers to screen the high risk 3-month major PSD group for early identification and intervention, using a prospective cohort of acute ischemic stroke (AIS) patients. Furthermore, we intended to construct a convenient screening nomogram for clinical use, integrating the associated sociodemographic, clinical factors and biomarkers of 3-month major PSD.

\section{Patients and Methods Study Population and Design}

A prospective cohort study was conducted in Tongji hospital, Wuhan Central Hospital and Wuhan First Hospital which all located in Wuhan City, Hubei Province, China. All procedures performed in studies involving human participants were in accordance with the 1964 Helsinki Declaration and its later amendments or comparable ethical standards. Informed consent was obtained from all individual participants included in the study. The registration number of this cohort study was ChiCTR-ROC -17013993. The original protocols used for this post hoc analysis did not include serum calcium, kalium, phosphorus and sodium as potential predictors.

Between May 2018 and August 2019, consecutively admitted AIS patients were recruited on admission and followed-up 3 months after the stroke onset. The inclusion criteria were as follows: i) males and females, aged $\geq 18$ years old, AIS patients diagnosed by brain magnetic resonance imaging (MRI) scans or computed tomography (CT) scans; ii) hospitalized within 7 days after stroke onset; iii) written informed consent was provided by patients or their authorized family members. The exclusion criteria were as follows: i) brain dysfunction caused by other non-vascular causes, such as primary brain tumors, metastatic encephaloma, subdural hematoma, paralysis after seizures, brain trauma, etc.; ii) history of depression (previous treatment history or clinical diagnosis), dementia and/or other psychiatric illness; iii) communication problems due to aphasia, severe dysarthria, disturbance of understanding or consciousness (a Mini-Mental State Examination score $<19$ points, in particular the MMSE score of illiterate patients was $<17$ points); ${ }^{21}$ iv) unable to complete the follow-up; v) transient ischemic attack (TIA) and subarachnoid hemorrhage; vi) with other concomitant neuropsychiatric diseases, such as Parkinson's disease and epilepsy; vii) without laboratory test for serum calcium or phosphorus on admission.

Serum samples were collected at room temperature on admission, then centrifuged at $3500 \mathrm{r} / \mathrm{min}$ for 10 minutes, which could be used to measure the levels of serum biomarkers. Depressive symptoms were measured at 3 months ( \pm 14 days) after onset of stroke, while the baseline 
information collection and routine laboratory tests were executed on admission.

\section{Primary Outcome}

The AIS patients were examined at a follow-up visit 3 months after the onset of stroke according to DSM-V criteria. The assessment was conducted in a quiet room between 9:00 am to $12: 00$ or $14: 00 \mathrm{pm}$ to $17: 00$ in the hospital. The timing of the assessment was also chosen to avoid the period of transient emotional adjustment to the disability caused by the acute ischemic stroke. ${ }^{22}$ All psychological evaluations were performed by three experienced neurologists after receiving standardized training for the neuropsychological assessments. The inter-rater reliability reached an acceptable level. The Hamilton Rating Scale for Depression-17 (HRSD-17) was used to measure the severity of 3 -month PSD. ${ }^{10}$ With major depression criteria being met in the DSM-V, HRSD score $\geq 17$ at 3 months after stroke onset was regarded as the primary endpoint. According to the existence of 3-month major PSD, patients were divided into major PSD group and non-major PSD group. The validity and reliability of the Chinese version HRSD-17 had been proven in previous studies. $^{23}$

\section{Demographic, Clinical Characteristics and Laboratory Measurements}

Patients' demographic information was collected with a case report form (CRF) on admission, covering gender, age, body mass index (BMI), degree of education, marital status and vascular risk factors, including history of smoking, drinking, stroke, diabetes mellitus, hypertension, hyperlipidemia, coronary heart disease, atrial fibrillation (AF) and surgery. National Institutes of Health and Stroke Scale score (NIHSS) was performed by trained neurologists to assess stroke severity on admission. The same applied to modified Rankin Scale (mRS) score.

The concentrations of serum calcium $(\mathrm{Ca})$, phosphorus $(\mathrm{P})$, kalium $(\mathrm{K})$, sodium $(\mathrm{Na})$, chlorine $(\mathrm{Cl})$ were measured by standard autoanalyzer techniques with a Roche automatic analyzer (cobas c 701) in clinical labs of the three hospitals. The cytokines, including IL-1 $\beta$, IL-6, IL-10, IL18 , TNF- $\alpha$, IFN- $\gamma$ and BDNF were measured using a solidphase sandwich enzyme-linked immunosorbent assay kit (CUSABIO, China) according to the manufacturer's specifications in Kindstar Company, Wuhan.
To minimize assay variance, all samples were analyzed on the same day in duplicate in a random order by a technician blinded to the clinical diagnoses; the intraassay coefficients were $<5 \%$. Calcium-phosphorus product was calculated by the formula of serum Ca level (mmol/ L) $\times$ serum P level $(\mathrm{mmol} / \mathrm{L}) \times 4 \times 3.1 \times \mathrm{mg}^{2} / \mathrm{dL}^{2}$.

\section{Statistical Analyses}

Data were analyzed by a biostatistician blinded to the study. Results were exhibited as median (interquartile range, IQR) for continuous variables while categorical variables were expressed as numbers (percentages). The proportion of the missing values in our study was $2.78 \%$. We applied multiple imputation method to substitute the missing values which could reduce the bias and increase the statistical power. The multiple copies were created and the missing values were substituted with imputed data according to a suitable random sample from their predicted distribution. We used the R package "mice" to obtain 5 completed data sets.

A 2-step procedure was used to develop the 3-month major PSD prediction model. First, we selected the most strongly associated predictors from each subgroup of variables, using multivariable logistic regression model with backward stepwise selection, $P<0.05$ for entry, $P>0.1$ for removal. Second, the final model was selected from this set of variables and validated with backward stepwise selection in multivariable logistic regression model. One thousand bootstrap samples were drawn from the original sample, estimating the overfitting corrected regression coefficients from the final model and the overfitting corrected measures of the model's performance, using the $\mathrm{R}$ package "rms". These statistics may be considered as an estimate of the performance that is expected in future patients. In backward step, selection was based on Wald $x^{2}$ test of the individual predictors with $P$ values $<0.05$.

All analyses were conducted in the 5 completed data sets. The 5 sets of regression coefficients, the performance estimates, and their variances were pooled according to Rubin's rules to produce estimates and confidence intervals (CIs) that incorporate missing-data uncertainty. To construct an easy-to-use clinical tool, the regression coefficients of the associated factors from the final model were applied to construct the nomogram. The total scores were linked to the risk of PSD. The discrimination ability of nomogram was expressed with the concordance statistic (C-statistic) with a 95\% CI, which was equal to the area under the receiver operating characteristic curve (AUC). We identified the nomogram as a useful clinical tool for 
decision making, particularly when the C-statistic was higher than $0.70 .{ }^{24}$ The higher values indicate better discrimination. The calibration curve of the established nomogram describes the extent to which the predicted probabilities of PSD reflect the true probabilities of PSD. All statistical analysis was performed with SPSS for Windows, version 22.0 (SPSS Inc., Chicago, IL, USA) and $\mathrm{R}$ version 3.5.2 software (The $\mathrm{R}$ Foundation for Statistical Computing, Vienna, Austria. http://www.r-pro ject.org). $P<0.05$ was set as significance level in twotailed significance values. The $\mathrm{R}$ packages "mice", "lattice”, "MASS", “nnet”, “cmprsk", "rms”, "Hmisc”, "Formula", and "ggplot2" were applied in data analysis.

\section{Results}

\section{Demographic, Laboratory, and Clinical Characteristics of the Study Subjects}

A total of 639 AIS patients, spanning the period between May 2018 and August 2019, who met the eligibility criteria were prospectively enrolled. 7.04\% (45/639) of patients were established as having 3-month major PSD. The sociodemographic, laboratory and clinical characteristics of the study subjects with and without major PSD were summarized in Table 1. The mean age was 63 (IQR, $56.5,69)$ years old in the major PSD patients group, 61 (IQR, 53, 68) in the non-major PSD patients group. Females accounted for $22.22 \%$ in non-major PSD patients group, $37.78 \%$ in major PSD patients group.

\section{Establishment and Validation of Nomogram in Predicting 3-Month Major PSD}

To determine the independent variables associated with 3-month major PSD, multivariate logistic regression analysis was performed. Variables with statistically significant differences in the results, including age, NIHSS score, mRS score, serum calcium-phosphorus product, educational level, history of hypertension and atrial fibrillation were identified between the 3-month major PSD and nonmajor PSD groups (all $P$ values $<0.05$ ). The results were presented in Table 2.

A nomogram to predict 3-month major PSD was developed based on multivariate logistic regression analysis using the 7 associated factors. The odds ratios for 3-month major PSD were calculated for these factors. As shown in Figure 1, there were 10 rows in the nomogram, with the rows ranging from 2 to 8 representing the selected variables. The points of the 7 variables were added up to the total points, which were displayed in row 9 and corresponded to the risk probability in the prediction of 3-month major PSD in row 10, and the nomogram showed the risk of major 3-month PSD as a percentage. A C-statistic of 0.81 (95\% CI, 0.791-0.829) for the nomogram was obtained, indicating the established nomogram performed well. The sensitivity and specificity were $71.1 \%$ and $78.6 \%$, respectively. The calibration plots with 1000 Bootstrap resamples were illustrated in Figure 2, showing that the nomogram-predicted probabilities of 3-month major PSD were similar to the actual probabilities of 3-month major PSD, which indicated that the nomogram was well calibrated.

\section{Discussion}

This was a multicenter prospective cohort study for PSD with large sample size. The three large general 3A hospitals in central China had covered a wide range of AIS patients with a big variation in functional status and thus produced a representative sample. In this study, we developed and validated a 3-month major PSD nomogram to identify those who are at high risk for 3-month major PSD based on effective predictors, which can be used to optimize patient care and follow-up in clinical practice. Although some sociodemographic, clinical factors and biomarkers have been found to be associated with PSD in previous studies, ${ }^{16,25-28}$ it is difficult to achieve an ideal precise prediction using a single indicator unless the combination of several effective predictors was applied, such as a nomogram. The nomogram showed good discrimination (C-statistic $=0.81,95 \% \mathrm{CI}, 0.791-0.829)$ and calibration through internal validation, which could prevent overfitting. Moreover, multiple imputation was regarded as the best method available to deal with both random and non-random missing values. ${ }^{29}$ The missing data in our study were imputed using multivariate imputation to create 5 imputed datasets which were pooled together into the final complete dataset, preventing biased estimates of the regression coefficients and their Standard Errors (SEs). ${ }^{17}$ These predictors, which can be easily obtained in clinical settings, ${ }^{30}$ will enhance the clinical utility of the prediction model in daily clinical practice.

Age and stroke severity (NIHSS score) have been considered as predictors of PSD in many studies. Some studies also reported that older age was a significant risk factor for PSD. ${ }^{31,32}$ Older patients dealing with the stress of acute stroke were more likely to have a depressive 
Table I Baseline Characteristics in Patients with and without Major PSD at 3 Months After Stroke Onset

\begin{tabular}{|c|c|c|}
\hline Patients & Non-Major PSD & Major PSD \\
\hline $\mathrm{N}$ & 594 & 45 \\
\hline Age, median (IQR, years) & 61 (53-68) & $63(56.5-69)$ \\
\hline Females, n (\%) & $132(22.22)$ & $17(37.78)$ \\
\hline BMI, median (IQR) & $24.22(22.49-26.70)$ & $24.22(21.75-26.78)$ \\
\hline \multicolumn{3}{|l|}{ Marital status, n (\%) } \\
\hline Single & $34(5.72)$ & $2(4.44)$ \\
\hline Married & $560(94.28)$ & $43(95.56)$ \\
\hline \multicolumn{3}{|l|}{ Educational level, n (\%) } \\
\hline Junior high school or below & $360(60.61)$ & $36(80.00)$ \\
\hline Senior high school or above & $234(39.39)$ & $9(20.00)$ \\
\hline NIHSS score, median (IQR) & $2(1,5)$ & $6(3,9)$ \\
\hline mRS score, median (IQR) & $2(1,3)$ & $4(2,4)$ \\
\hline \multicolumn{3}{|c|}{ Vascular risk factors and medical history, $\mathrm{n}(\%)$} \\
\hline History of smoking & $357(60.10)$ & $19(42.22)$ \\
\hline History of drinking & $322(54.21)$ & $25(55.56)$ \\
\hline History of diabetes mellitus & $161(27.10)$ & $9(20.00)$ \\
\hline History of hypertension & $357(60.10)$ & $23(5 \mathrm{I} .1 \mathrm{I})$ \\
\hline History of hyperlipidemia & $139(23.40)$ & II (24.44) \\
\hline History of coronary heart disease & $55(9.26)$ & $4(8.89)$ \\
\hline History of stroke & $125(21.04)$ & $7(15.56)$ \\
\hline History of atrial fibrillation & $166(27.95)$ & $5(11.11)$ \\
\hline History of surgery & $18(3.03)$ & $14(31.11)$ \\
\hline \multicolumn{3}{|l|}{ Laboratory findings, median (IQR) } \\
\hline Calcium-phosphorus product & $29.16(25.29,33.25)$ & $29.46(27.28,35.00)$ \\
\hline Kalium (mmol/L) & $3.90(3.66,4.15)$ & $3.90(3.60,4.24)$ \\
\hline Sodium (mmol/L) & $140.8(139.1,142.0)$ & $140.0(|37.4| 4 \mid .9)$, \\
\hline Chlorine $(\mathrm{mmol} / \mathrm{L})$ & $103.2(101.0,105.3)$ & $101.7(99.95,103.85)$ \\
\hline IL-I $\beta$, median (IQR, pg/mL) & $66.01(27.37,133.15)$ & $63.59(28.17,174.84)$ \\
\hline IL-6, median (IQR, pg/mL) & $6.00(1.83,13.94)$ & $6.00(3.06,9.90)$ \\
\hline IL-I0, median (IQR, ng/L) & $7.30(3.02,16.96)$ & $9.74(3.44,40.74)$ \\
\hline TNF- $\alpha$, median (IQR, pg/mL)) & $40.23(17.56,61.46)$ & $43.12(23.77,60.37)$ \\
\hline BDNF, median (IQR, ng/mL) & $2.67(1.54,5.67)$ & $3.82(2.19,7.44)$ \\
\hline IFN- $\gamma$, median (IQR, pg/mL) & $4.34(2.22,10.02)$ & $4.42(1.65,9.74)$ \\
\hline IL-I8, median (IQR, pg/mL)) & $1549.00(796.09,6416.13)$ & $1979.36(931.58,4432.86)$ \\
\hline
\end{tabular}

response, possibly due to lack of sufficient social security. ${ }^{33}$ Higher NIHSS score was associated with 3-month major PSD because the severity of neurological impairment in AIS patients probably impacted on their quality of life, caused great psychological pressure and negative emotions. ${ }^{11}$ A previous study also reported that baseline mRS score $>2$ was associated with two fold increased risk for depression. ${ }^{34}$ Additionally, the Secondary Prevention of Small Subcortical Strokes (SPS3) study showed that patients with mRS of 2-3 were twice as likely to develop depressive symptoms. ${ }^{35}$ A systematic review also confirmed that depression was negatively associated with functional outcome in stroke survivors, both acutely and later after stroke. ${ }^{36}$ The focus of this study was on a novel marker, serum calciumphosphorus product, which has not been examined previously in relation to PSD. The elevated levels of serum calcium-phosphorus product were associated with increased risk of major PSD at 3 months, and this correlation was still significant after adjusting for potential risk 
Table 2 Signifciant Variables Associated with 3-Month Major PSD by Multivariable Logistic Regression

\begin{tabular}{|l|c|c|c|c|}
\hline Variables & Odds Ratio $\mathbf{( 9 5 \% ~ C l )}$ & Coefficient & SE & P value \\
\hline Calcium-phosphorus product & $1.080(1.029-1.133)$ & 0.077 & 0.025 & 0.002 \\
Age & $1.049(1.013-1.086)$ & 0.048 & 0.018 & 0.007 \\
Educational level & $0.384(0.174-0.847)$ & -0.958 & 0.404 & 0.018 \\
History of hypertension & $0.446(0.227,0.875)$ & -0.808 & 0.344 & 0.019 \\
History of atrial fibrillation & $4.416(1.412-13.810)$ & 1.485 & 0.582 & 0.011 \\
NIHSS score & $1.149(1.030-1.282)$ & 0.139 & 0.056 & 0.013 \\
mRS score & $1.559(1.127-2.157)$ & 0.444 & 0.166 & 0.007 \\
\hline
\end{tabular}

factors in 5 multiple imputation datasets. The results suggested that calcium-phosphorus product was possibly a potential biomarker in prediction of 3-month major PSD. Previous studies reported that calcium-phosphorus product was an effective marker of subclinical carotid atherosclerosis, ${ }^{37}$ or atherosclerosis in adults with chronic kidney disease, such as increased carotid intima-media thickness (IMT) and coronary calcification. ${ }^{38,39}$ Zhang et al reported that carotid stenosis was a possible independent risk factor of PSD. ${ }^{11}$ The vascular depression hypothesis proposes that cerebrovascular disease predisposes, precipitates, and perpetuates a late-life depression syndrome. The association between calcium-phosphorus product and 3-month major PSD may be mediated by carotid stenosis.

The increased risk of PSD was associated with history of atrial fibrillation, which could be explained by the higher recurrent risk of stroke caused by atrial fibrillation, especially in cardioembolic stroke. ${ }^{40,41}$ Hypertension may then not only relate to risk of stroke, and possibly to stroke severity, but also contribute to the risk of depression following stroke through the development of small-vessel brain disease, resulting in damage to specific brain circuits involved in mood. Hypertension may also relate to depression less directly, through inflammation or other biological mechanisms. ${ }^{42}$ Another link may stem from the fact that some medications used to treat hypertension have been linked to depression. ${ }^{43}$ Moreover, patients with a previous history of hypertension may accept the presence of hypertension and its possible role in the pathogenesis of stroke as well as its associated disability. These patients may have better psychological coping mechanism after the occurrence of stroke compared to the patients without a history of hypertension who did not expect any stroke disability. ${ }^{44}$ Higher educational level appeared to be a factor associated with major PSD at 3 months, probably because it was associated with better functional cognitive reserve. ${ }^{45}$

The main strengths of this study were the following: multicenter prospective cohort with enrollment of sequential AIS cases; complete radiological and clinical evaluations;

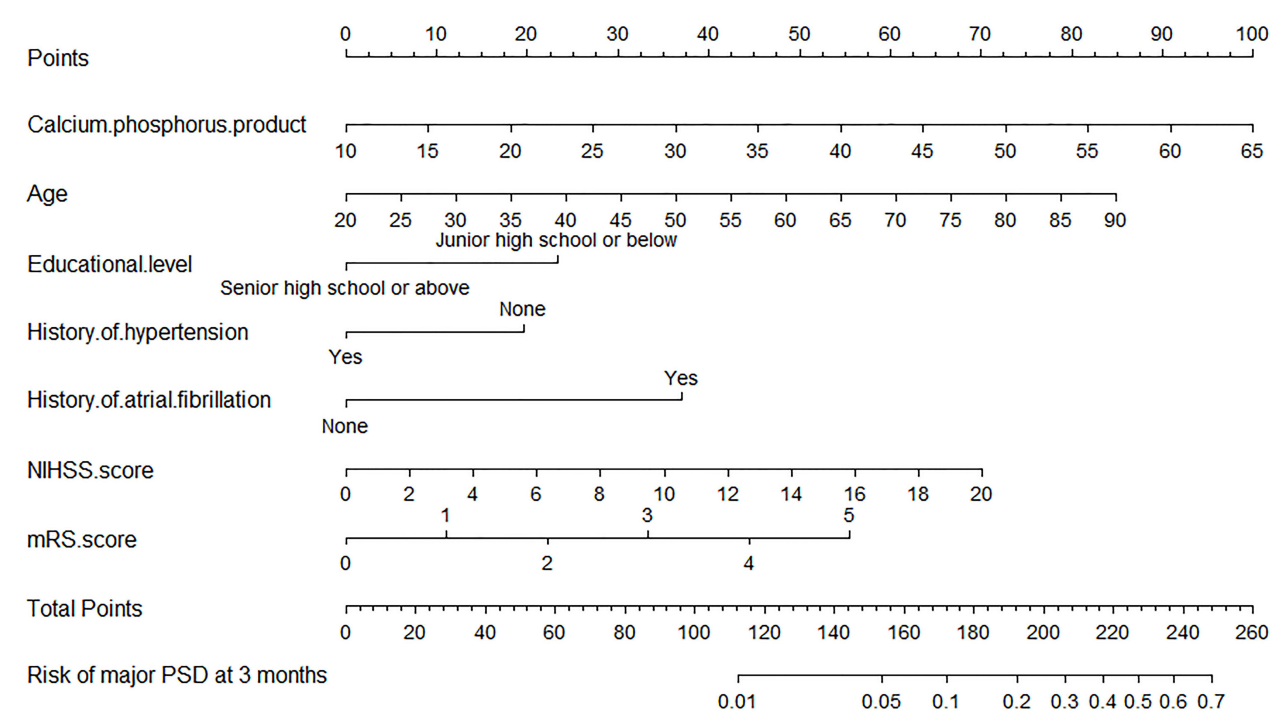

Figure I Nomograms predicting 3-month major PSD. 


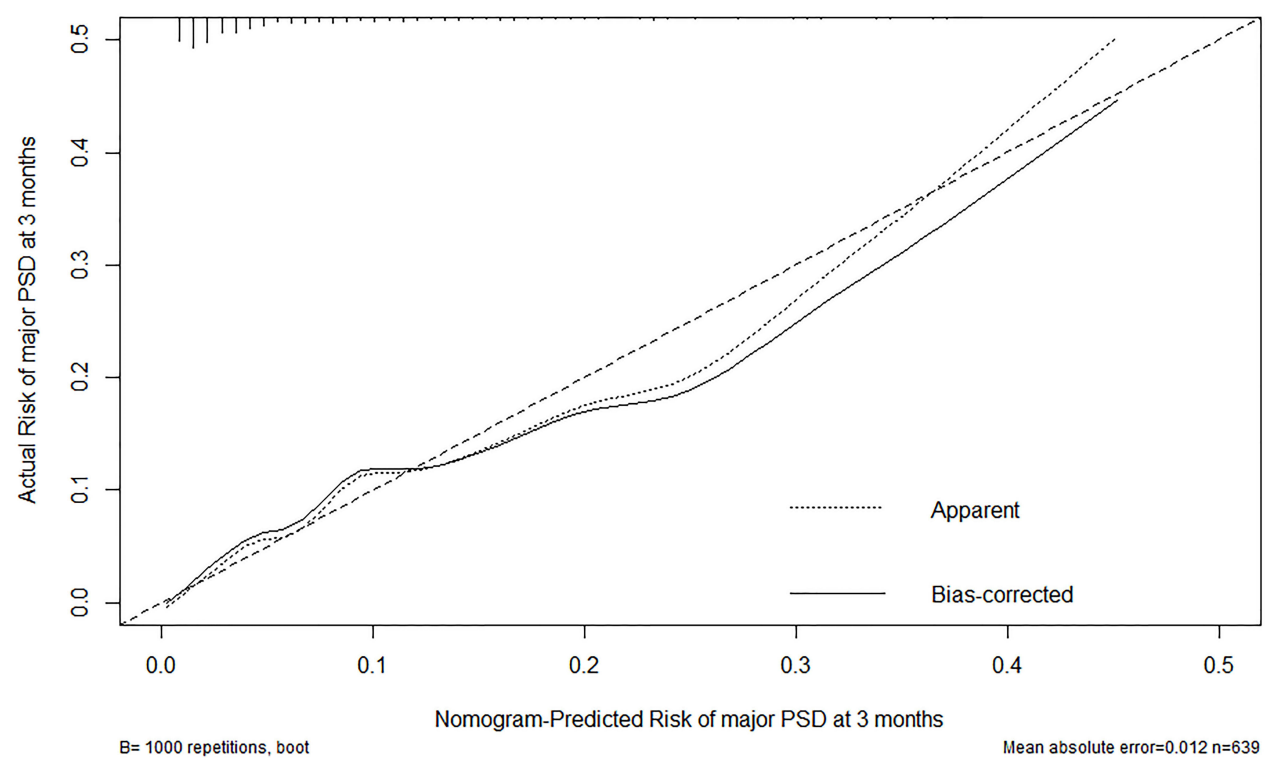

Figure 2 Calibration plots of the nomogram predicting 3-month major PSD.

systematic neuropsychological assessment with a wide battery of tests with participation of a multidisciplinary team; practical screening nomogram was generated for clinical use. There were also several limitations that may hinder the generalization of nomograms. First, this was a hospital-based sample which inevitably could result in admission bias. Our preliminary clinical tool requires future confirmation studies in a larger community-based sample. Secondly, it did not include patients with more severe strokes who were unable to communicate effectively with the clinical staff due to aphasia and severe dysarthria. Research has shown that patients with more severe strokes have higher depression rates, thus our data likely underestimated the actual incidence of 3-month major PSD in our study. ${ }^{28}$ Thirdly, we did not determine the serum calcium-phosphorus product levels during follow-up, so further studies are needed to assess how calcium-phosphorus product levels change with time after stroke, and to evaluate the diagnostic value of calcium-phosphorus product comprehensively. It is necessary to compare the results of laboratory tests after 3 months. Fourthly, this was an observational cohort study which could not draw any causal relationship. This was a preliminary study with a small sample $(\mathrm{N}=639)$ with a few 3-month major PSD patients $(\mathrm{N}=45)$ and all the participants were Chinese. The conclusion should not be overinterpreted.

\section{Conclusion}

In summary, we identified several associated factors that could predict 3-month major PSD. Notably, our findings demonstrated the association between the calcium-phosphorus product levels on admission and the development of 3-month major PSD. The findings should be considered as preliminary, and further community-based trials are needed to investigate the diagnostic value of calcium-phosphorus product for 3-month major PSD, which may provide a new proposal for new therapeutic options. In addition, we developed a nomogram for predicting the risk of 3-month major depression in AIS patients. The nomogram was easy to operate with good discrimination and calibration. This nomogram could be used as a convenient screening tool in clinical practice to guide follow-up and aid accurate prognostic assessment. The nomogram should also be validated in other AIS patients.

\section{Data Sharing Statement}

The data and $R$ codes that were used to generate the results can be made available upon request from corresponding authors.

\section{Ethics Approval and Informed Consent}

The protocol was approved by the Ethics Committee of Tongji Medical College, Huazhong University of Science and Technology (ID: TJ-IRB20171108). Written informed consent was obtained from all subjects or their caregivers.

\section{Author Contributions}

All authors met the following conditions: 
1. Made a significant contribution to the work reported, whether that is in the conception, study design, execution, acquisition of data, analysis and interpretation, or in all these areas.

2. Drafted or wrote, or substantially revised or critically reviewed, the article.

3. Agreed on the journal to which the article will be submitted.

4. Reviewed and agreed on all versions of the article before submission, during revision, the final version accepted for publication, and any significant changes introduced at the proofing stage.

5. Agreed to take responsibility and be accountable for the contents of the article.

\section{Funding}

This work was financially supported by the National Key Research \& Development Program of China [grant number 2017YFC1310000], Hubei Technological Innovation Special Fund [grant number 2019ACA132]. The funders had no role in study design, data collection and analysis, decision to publish.

\section{Disclosure}

All authors declare no competing interests in this work.

\section{References}

1. Feigin VL, Nguyen G, Cercy K, et al. Global, Regional, and Country-Specific Lifetime Risks of Stroke, 1990 and 2016. N Engl J Med. 2018;379(25):2429-2437.

2. Robinson RG, Jorge RE. Post-Stroke Depression: a Review. Am $J \quad$ Psychiatry. 2016;173(3):221-231. doi:10.1176/appi. ajp.2015.15030363

3. Flaster M, Sharma A, Rao M. Poststroke depression: a review emphasizing the role of prophylactic treatment and synergy with treatment for motor recovery. Top Stroke Rehabil. 2013;20(2):139-150. doi:10.1310/tsr2002-139

4. Ayerbe L, Ayis S, Crichton S, et al. The natural history of depression up to 15 years after stroke: the South London Stroke Register. Stroke. 2013;44(4):1105-1110. doi:10.1161/STROKEAHA.111.679340

5. Whyte EM, Mulsant BH. Post stroke depression: epidemiology, pathophysiology, and biological treatment. Biol Psychiatry. 2002;52 (3):253-264. doi:10.1016/S0006-3223(02)01424-5

6. Chemerinski E, Robinson RG, Kosier JT. Improved recovery in activities of daily living associated with remission of poststroke depression. Stroke. 2001;32(1):113-117. doi:10.1161/01.STR.32.1.113

7. Bour A, Schepers VPM, Nijsse B, van Heugten CM, Post MWM, Visser-Meily JMA. A one-year follow-up study into the course of depression after stroke. J Nutr Health Aging. 2010;14(6):488-493. doi:10.1007/s12603-010-0033-x

8. Bour A, Rasquin S, Limburg M, Verhey F. Depressive symptoms and executive functioning in stroke patients: a follow-up study. Int J Geriatr Psychiatry. 2011;26(7):679-686. doi:10.1002/gps.2581
9. Yang Y, Shi YZ, Zhang N, et al. The Disability Rate of 5-Year Post-Stroke and Its Correlation Factors: a National Survey in China. PLoS One. 2016;11(11):e0165341. doi:10.1371/journal. pone. 0165341

10. Huang J, Zhou FC, Guan B, et al. Predictors of Remission of Early-Onset Poststroke Depression and the Interaction Between Depression and Cognition During Follow-Up. Front Psychiatry. 2018;9:738. doi:10.3389/fpsyt.2018.00738

11. Zhang Y, Cheng L, Chen Y, Yang GY, Liu J, Zeng L. Clinical predictor and circulating microRNA profile expression in patients with early onset post-stroke depression. $J$ Affect Disord. 2016;193:51-58. doi:10.1016/j.jad.2015.12.061

12. Huang G, Chen H, Wang Q, et al. High platelet-to-lymphocyte ratio are associated with post-stroke depression. $J$ Affect Disord. 2019;246:105-111. doi:10.1016/j.jad.2018.12.012

13. Tu WJ, Dong X, Zhao SJ, Yang DG, Chen H. Prognostic value of plasma neuroendocrine biomarkers in patients with acute ischaemic stroke. J Neuroendocrinol. 2013;25(9):771-778. doi:10.1111/jne.12052

14. Lu X, Duan J, Cheng Q, Lu J. The association between serum growth differentiation factor-15 and 3-month depression after acute ischemic stroke. J Affect Disord. 2020;260:695-702. doi:10.1016/j.jad.2019.09.037

15. Wang G, Zhou Y, Bu X, et al. Antiphospholipid antibodies predict post-stroke depression after acute ischemic stroke. $J$ Affect Disord. 2019;257:160-165. doi:10.1016/j.jad.2019.07.013

16. Tu WJ, Qiu HC, Liu Q, et al. Decreased level of irisin, a skeletal muscle cell-derived myokine, is associated with post-stroke depression in the ischemic stroke population. J Neuroinflammation. 2018;15 (1):133. doi:10.1186/s12974-018-1177-6

17. Zhao H, Mo M, Miao C, et al. Association of serum biomarker neurofilament light concentration with post-stroke depression: a preliminary study. Gen Hosp Psychiatry. 2020;64:17-25. doi:10.1016/j.genhosppsych.2020.01.006

18. de Man-van GJ, Hafsteinsdottir TB, Lindeman E, et al. In-hospital risk prediction for post-stroke depression: development and validation of the Post-stroke Depression Prediction Scale. Stroke. 2013;44 (9):2441-2445. doi:10.1161/STROKEAHA.111.000304

19. Li G, Mei J, You J, et al. Sociodemographic characteristics associated with adolescent depression in urban and rural areas of Hubei province: a cross-sectional analysis. BMC Psychiatry. 2019;19(1):386. doi:10.1186/s12888-019-2380-4

20. Li G, Lian L, Huang S, et al. Nomograms to predict 2-year overall survival and advanced schistosomiasis-specific survival after discharge: a competing risk analysis. J Transl Med. 2020;18(1):187. doi:10.1186/s12967-020-02353-5

21. Katzman R, Zhang MY, Qu O-Y, et al. A Chinese version of the Mini-Mental State Examination; impact of illiteracy in a Shanghai dementia survey. $J$ Clin Epidemiol. 1988;41(10):971-978. doi:10.1016/0895-4356(88)90034-0

22. Tang WK, Chen YK, Lu JY, et al. Cerebral microbleeds and depression in lacunar stroke. Stroke. 2011;42(9):2443-2446. doi:10.1161/ STROKEAHA.111.614586

23. Zheng YP, Zhao JP, Phillips M, et al. Validity and reliability of the Chinese Hamilton Depression Rating Scale. $\mathrm{Br} J$ Psychiatry. 1988;152:660-664. doi:10.1192/bjp.152.5.660

24. James MT, Pannu N, Hemmelgarn BR. Derivation and External Validation of Prediction Models for Advanced Chronic Kidney Disease Following Acute Kidney Injury. JAMA. 2017;318 (18):1787-1797. doi:10.1001/jama.2017.16326

25. Pascoe MC, Crewther SG, Carey LM, Noonan K, Crewther DP, Linden T. Homocysteine as a potential biochemical marker for depression in elderly stroke survivors. Food Nutr Res. 2012;56. doi:10.3402/fnr.v56i0.14973

26. Xiao M, Wang Q, Ren W, et al. Impact of prediabetes on poststroke depression in Chinese patients with acute ischemic stroke. Int J Geriatr Psychiatry. 2018;33(7):956-963. doi:10.1002/gps.4878 
27. Kim JM, Kang HJ, Kim JW, et al. Associations of Tumor Necrosis Factor-alpha and Interleukin-1beta Levels and Polymorphisms with Post-Stroke Depression. Am J Geriatr Psychiatry. 2017;25 (12):1300-1308. doi:10.1016/j.jagp.2017.07.012

28. Liu Z, Zhu Z, Zhao J, et al. Malondialdehyde: a novel predictive biomarker for post-stroke depression. $J$ Affect Disord. 2017;220:95-101.

29. Donders AR, van der Heijden GJ, Stijnen T, Moons KG. Review: a gentle introduction to imputation of missing values. $J$ Clin Epidemiol. 2006;59(10):1087-1091. doi:10.1016/j.jclinepi.2006.01.014

30. Li G, Huang S, Lian L, et al. Derivation and external validation of a model to predict 2-year mortality risk of patients with advanced schistosomiasis after discharge. EBioMedicine. 2019;47:309-318. doi:10.1016/j.ebiom.2019.08.028

31. Linden T, Blomstrand C, Skoog I. Depressive disorders after 20 months in elderly stroke patients: a case-control study. Stroke. 2007;38(6):1860-1863. doi:10.1161/STROKEAHA.106.471805

32. Kauhanen M, Korpelainen JT, Hiltunen P, et al. Poststroke depression correlates with cognitive impairment and neurological deficits. Stroke. 1999;30(9):1875-1880. doi:10.1161/01.STR.30.9.1875

33. De Ryck A, Brouns R, Geurden M, et al. Risk factors for poststroke depression: identification of inconsistencies based on a systematic review. J Geriatr Psychiatry Neurol. 2014;27(3):147-158. doi:10.1177/0891988714527514

34. Pavlovic AM, Pekmezovic T, Zidverc Trajkovic J, et al. Baseline characteristic of patients presenting with lacunar stroke and cerebral small vessel disease may predict future development of depression. Int $J$ Geriatr Psychiatry. 2016;31(1):58-65. doi: $10.1002 /$ gps.4289

35. White CL, McClure LA, Wallace PM, et al. The correlates and course of depression in patients with lacunar stroke: results from the Secondary Prevention of Small Subcortical Strokes (SPS3) study. Cerebrovasc Dis. 2011;32(4):354-360.
36. Kutlubaev MA, Hackett ML, Part II. predictors of depression after stroke and impact of depression on stroke outcome: an updated systematic review of observational studies. Int J Stroke. 2014;9 (8):1026-1036. doi:10.1111/ijs.12356

37. Carrelli AL, Walker MD, Lowe H, et al. Vitamin D deficiency is associated with subclinical carotid atherosclerosis: the Northern Manhattan study. Stroke. 2011;42(8):2240-2245. doi:10.1161/ STROKEAHA.110.608539

38. Oh J, Wunsch R, Turzer M, et al. Advanced coronary and carotid arteriopathy in young adults with childhood-onset chronic renal failure. Circulation. 2002;106(1):100-105. doi:10.1161/01. CIR.0000020222.63035.C0

39. Raggi P, Boulay A, Chasan-Taber S, et al. Cardiac calcification in adult hemodialysis patients. A link between end-stage renal disease and cardiovascular disease? J Am Coll Cardiol. 2002;39(4):695-701. doi:10.1016/S0735-1097(01)01781-8

40. Hankey GJ. Stroke. Lancet. 2017;389(10069):641-654. doi:10.1016/ S0140-6736(16)30962-X

41. Wang Y, Qian Y, Smerin D, et al. Newly Detected Atrial Fibrillation after Acute Stroke: a Narrative Review of Causes and Implications. Cardiology. 2019;144(3-4):112-121. doi:10.1159/000502971

42. Alexopoulos GS, Kelly RJ. Research advances in geriatric depression. World Psychiatry. 2009;8(3):140-149. doi:10.1002/ j.2051-5545.2009.tb00234.x

43. Beers MH, Passman LJ. Antihypertensive medications and depression. Drugs. 1990;40(6):792-799. doi:10.2165/00003495199040060-00003

44. Wongwandee M, Tangwongchai S, Phanthumchinda K. Relationship between poststroke depression and ischemic lesion location. $J \mathrm{Med}$ Assoc Thai. 2012;95(3):330-336.

45. Arauz A, Rodríguez-Agudelo Y, Sosa AL, et al. Vascular cognitive disorders and depression after first-ever stroke: the Fogarty-Mexico Stroke Cohort. Cerebrovasc Dis. 2014;38(4):284-289. doi:10.1159/000366471
Clinical Interventions in Aging

\section{Publish your work in this journal}

Clinical Interventions in Aging is an international, peer-reviewed journal focusing on evidence-based reports on the value or lack thereof of treatments intended to prevent or delay the onset of maladaptive correlates of aging in human beings. This journal is indexed on PubMed Central, MedLine, CAS, Scopus and the Elsevier

\section{Dovepress}

Bibliographic databases. The manuscript management system is completely online and includes a very quick and fair peer-review system, which is all easy to use. Visit http://www.dovepress.com/ testimonials.php to read real quotes from published authors. 